\title{
Isospin symmetry breaking and Baryon-Isospin correlations in effective mean field models.
}

\author{
Abhijit Bhattacharyya \\ University of Calcutta, Kolkata \\ E-mail: abphy@caluniv.ac.in

\section{Sanjay K. Ghosh} \\ Center for Astroparticle Physics \& Space Science, Bose Institute, Kolkata \\ E-mail: sanjayajcbose.ac.in
}

\section{Anirban Lahiri}

Tata Institute of Fundamental Research, Mumbai

E-mail: anirbanlahiri.physics@gmail.com

\section{Sarbani Majumder*}

Saha Institute of Nuclear Physics, Kolkata, India.

E-mail: sarbanimajumder@gmail.com

\section{Sibaji Raha}

Center for Astroparticle Physics \& Space Science, Bose Institute, Kolkata E-mail: sibajiejcbose.ac.in

\section{Rajarshi Ray}

Center for Astroparticle Physics \& Space Science, Bose Institute, Kolkata

E-mail: rajarshi@jcbose.ac.in

\begin{abstract}
A study of the $1+1$ flavor system of strongly interacting matter in terms of the Polyakov-Nambu-Jona-Lasinio model is presented. It is found that though the small isospin symmetry breaking brought in through unequal light quark masses is too small to affect the thermodynamics of the system in general, it may have significant effect in baryon-isospin correlations and have a measurable impact in heavy-ion collision experiments.
\end{abstract}

7th International Conference on Physics and Astrophysics of Quark Gluon Plasma

1-5 February, 2015

Kolkata, India

$$
\text { *speaker }
$$




\section{Introduction}

Quantum Chromodynamics (QCD) is the formulation for first principle studies of strongly interacting matter. Along with the local color symmetry, the quark sector has few global symmetries also. In the chiral limit for two light flavors $u$ and $d$, we have global vector and axial vector symmetry $S U_{V}(2) \otimes S U_{A}(2)$. For non-zero quark masses, the axial symmetry $S U_{A}(2)$ is explicitly broken, while for non-zero quark mass difference vector (isospin) symmetry $S U_{V}(2)$ is explicitly broken. Apart from the quark mass difference, ISB effects may be brought in by electromagnetic contributions as well.

Some Lattice QCD investigation of the effect of unequal quark masses was done in Ref.[1]. Recently in Ref.[2,3] the effect of ISB on different hadronic observables were studied. Within the framework of chiral perturbation theory the isospin breaking effect in quark condensates has been studied considering $m_{u} \neq m_{d}$ and electromagnetic corrections as well, where an analysis of scalar susceptibilities $[4,5]$ is given. In effective mean field model like Nambu-Jona-Lasinio (NJL) model, both of the above-mentioned effects have been incorporated [6] to study the influence of the isospin symmetry breaking on the orientation of chiral symmetry breaking. In the present work we describe the first case study of ISB effect on fluctuations and correlations of strongly interacting matter within the framework of the Polyakov loop enhanced Nambu-Jona-Lasinio (PNJL) model. We discuss the possible experimental manifestations of the ISB effects based on quite general considerations in the limit of small current quark masses.

\section{Formalism}

Here we use the form of the 2 flavor PNJL model with the Lagrangian as in Ref.[7, 8];

$$
\begin{aligned}
\mathscr{L}_{P N J L}= & -\mathscr{U}[\Phi[A], \bar{\Phi}[A], T]+\bar{\psi}(\not D-\hat{m}) \psi \\
& +G_{1}\left[(\bar{\psi} \psi)^{2}+(\bar{\psi} \vec{\tau} \psi)^{2}+\left(\bar{\psi} i \gamma_{5} \psi\right)^{2}+\left(\bar{\psi} i \gamma_{5} \vec{\tau} \psi\right)^{2}\right] \\
& +G_{2}\left[(\bar{\psi} \psi)^{2}-(\bar{\psi} \vec{\tau} \psi)^{2}-\left(\bar{\psi} i \gamma_{5} \psi\right)^{2}+\left(\bar{\psi} i \gamma_{5} \vec{\tau} \psi\right)^{2}\right]
\end{aligned}
$$

$\mathscr{U}[\Phi[A], \bar{\Phi}[A], T]$ is the effective potential expressed in terms of traced Polyakov loop $\Phi$ and its charge conjugate $\bar{\Phi}$. Here we shall consider a mass matrix of the form:

$$
\begin{aligned}
\hat{m} & =m_{1} 11_{2 \times 2}-m_{2} \tau_{3} \\
& =\left(\begin{array}{cc}
m_{1}-m_{2} & 0 \\
0 & m_{1}+m_{2}
\end{array}\right) \equiv\left(\begin{array}{cc}
m_{u} & 0 \\
0 & m_{d}
\end{array}\right) .
\end{aligned}
$$

where, $11_{2 \times 2}$ is the identity matrix in flavor space and $\tau_{3}$ is the third Pauli matrix. Here $m_{u}$ and $m_{d}$ are the current masses of the $u$ and $d$ quarks respectively. While a non-zero $m_{1}$ breaks the chiral $S U_{A}(2)$ symmetry explicitly a non-zero $m_{2}$ does the same for the isospin $S U_{V}(2)$ symmetry. We have restricted ourselves to $G_{1}=G_{2}=G$ which implies $m_{2}=\left(M_{d}-M_{u}\right) / 2$, where $M_{u}$ and $M_{d}$ are the constituent masses of the $u$ and $d$ quarks respectively. Deriving the thermodynamic potential from Lagrangian, different charge susceptibilities can be obtained from corresponding chemical potential derivative of thermodynamic potential. 


\section{Off-diagonal Susceptibilities for $\mu_{B}=0$}

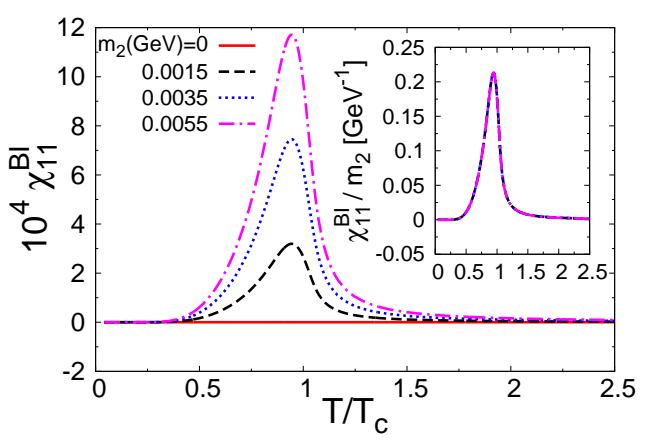

Figure 1: Second order off-diagonal susceptibility in $B-I$ sector at $\mu_{B}=0$.

In Fig. 1 the second order off-diagonal susceptibility $\chi_{11}^{B I}$, is shown for different values of $m_{2}$. As expected we find $\chi_{11}^{B I}=0$ for $m_{2}=0$. For non-zero $m_{2}$ we find $\chi_{11}^{B I}$ to have non-zero values that change non-monotonically with the increase in temperature.

An exciting feature observed here is that there is an almost linear scaling of $\chi_{11}^{B I}$ with $m_{2}$. This is shown in the inset of Fig.1.
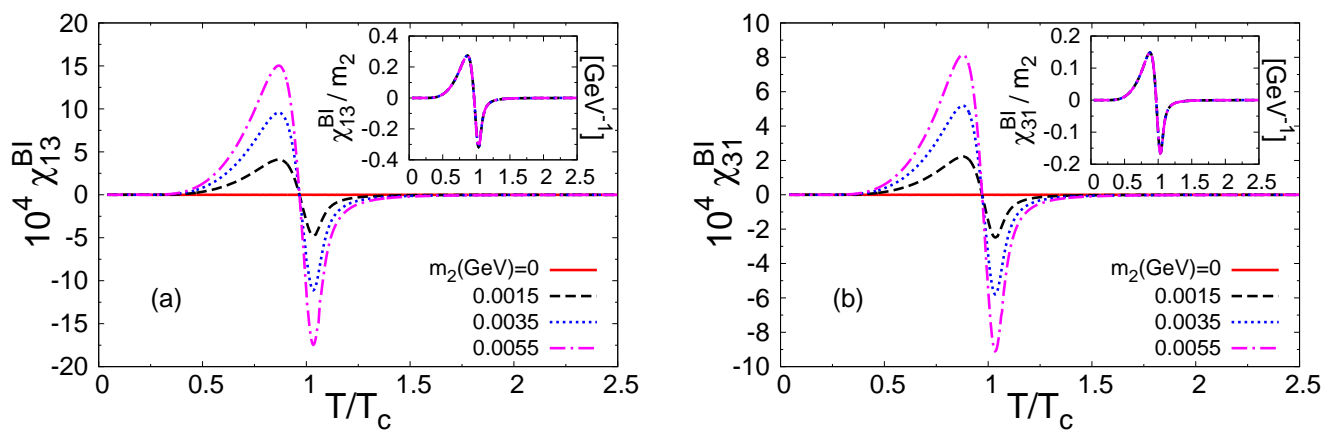

Figure 2: Behavior of 4th order off diagonal susceptibility for different $m_{2}$.

The fourth order off-diagonal susceptibilities in the $B-I$ sector are $\chi_{13}^{B I}, \chi_{31}^{B I}$ and $\chi_{22}^{B I}$. The $m_{2}$ dependence of $\chi_{22}^{B I}$ was found to be insignificant. For $\mu_{B}=0$, the $T$ dependence for the other two susceptibilities along with their $m_{2}$ scaling is shown in Fig.2.

We can express different $B-I$ correlators in terms of those in the flavor space. The flavor diagonal susceptibilities can be expanded in a Taylor series of the quark masses around $m_{u}=m_{d}=$ 0 . From this one is able to undestand the presence of $m_{2}$ scaling for some correlators. The detail analysis is shown in [9].

\section{Off diagonal Susceptibilities for $\mu_{B} \neq 0$}

Here we show the variation of $\chi_{11}^{B I}$ with $\mu_{B}$ for four different temperatures. The features vary widely over the different ranges of temperature and chemical potential. At $T \sim 2 T_{c}, \chi_{11}^{B I}$ is positive, and slowly decreases with increasing $\mu_{B}$. Close to $T_{c}, \chi_{11}^{B I}$ drops sharply to zero, becomes negative 
and then again slowly approaches zero. Going down somewhat below $T_{C}$ there is an initial increase in $\chi_{11}^{B I}$ for some range of $\mu_{B}$, and thereafter it follows the behavior at $T_{c}$. Finally at very low temperatures the change in sign of $\chi_{11}^{B I}$ is marked by a discontinuity, arising due to a first order phase boundary which exists in this range of $T$ and $\mu_{B}$.
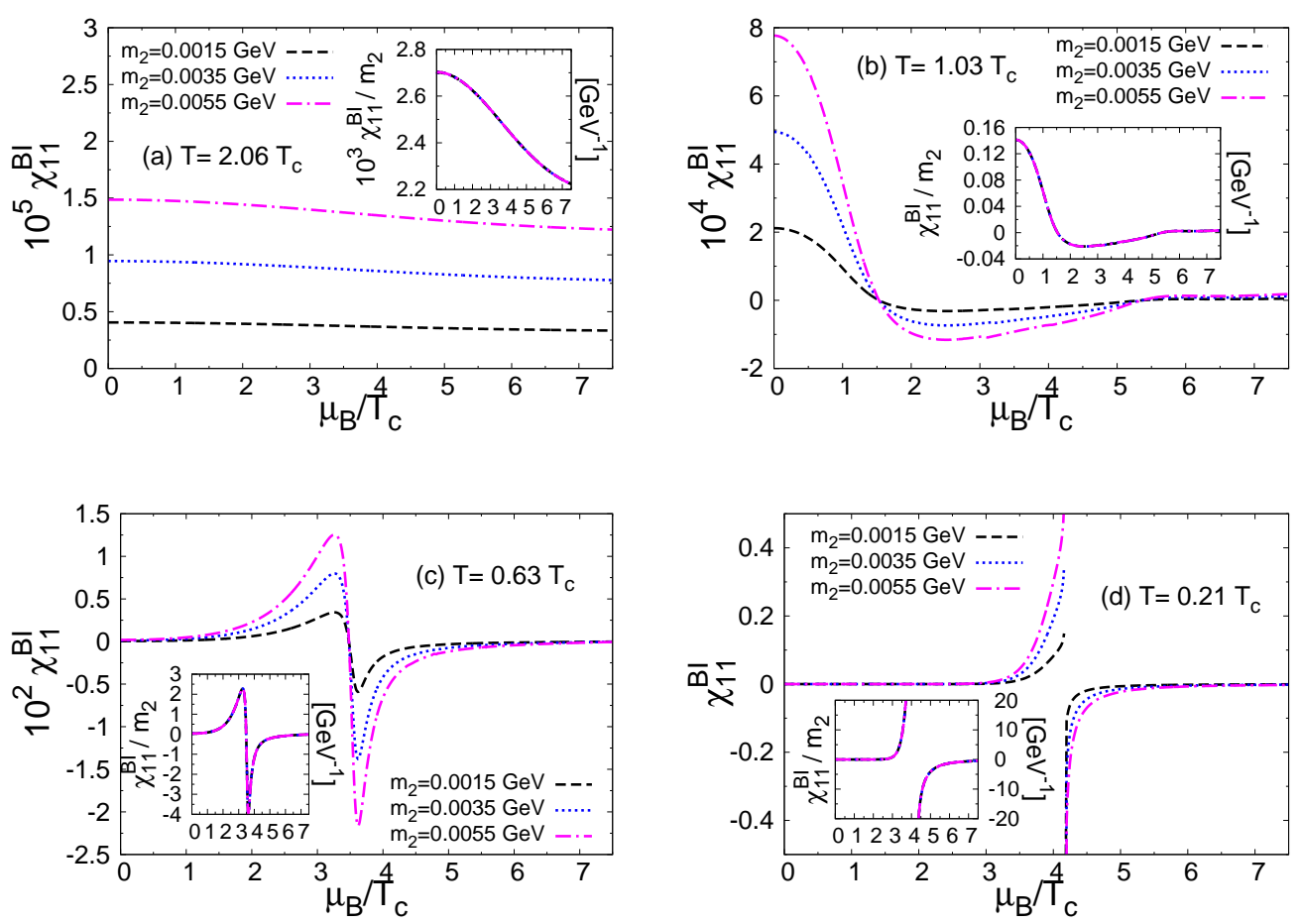

Figure 3: $\chi_{11}^{B I}$ along baryon chemical potential at different temperatures.

These various features can be understood by expressing $\chi_{11}^{B I}=\frac{\partial}{\partial \mu_{B}}\left(\frac{\partial P}{\partial \mu_{I}}\right)=\left(\frac{\partial n_{I}}{\partial \mu_{B}}\right)$, where $n_{I}$ is the isospin number density [9].

\section{Further implications of ISB in Heavy Ion Collisions}

Correlation between conserved charges, is an experimentally measurable quantity obtained from event-by-event analysis in heavy-ion collisions [10]. To compare with experiments it is often useful to consider ratios such as $\mathrm{R}_{2}=\chi_{11}^{B I} / \chi_{2}^{B}=\mathrm{C}_{B I} / \mathrm{C}_{B B}[10,11]$. Here $\mathrm{C}_{X Y}=\frac{1}{N_{E}} \sum_{i=1}^{N_{E}} X_{i} Y_{i}-$ $\left(\frac{1}{N_{E}} \sum_{i=1}^{N_{E}} X_{i}\right) \cdot\left(\frac{1}{N_{E}} \sum_{i=1}^{N_{E}} Y_{i}\right)$, where $N_{E}$ is the total number of events considered and $X_{i}$ and $Y_{i}$ are the event variables corresponding to the conserved charges in a given event $i$. Ratios of this kind are practically useful in eliminating uncertainties in the estimates of the measured volume of the fireball. The temperature variation of $\mathrm{R}_{2}$ obtained here is shown in Fig.4. It decreases monotonically and approaches zero above $T_{c}$. This is expected as the baryon number fluctuation increases much more rapidly than the $B-I$ correlation below $T_{c}$, and thereafter $\chi_{11}^{B I}$ goes to zero while $\chi_{2}^{B}$ attains a non-zero value. The $m_{2}$ scaling that we observed for $\chi_{11}^{B I}$ or $\mathrm{R}_{2}$ is most likely model independent as it is expected on very general grounds for small current quark masses as discussed above. Therefore, at any temperature and chemical potential, one can use the $m_{2}$ scaling to estimate the mass asymmetry of constituent fermions in a physical system as, 


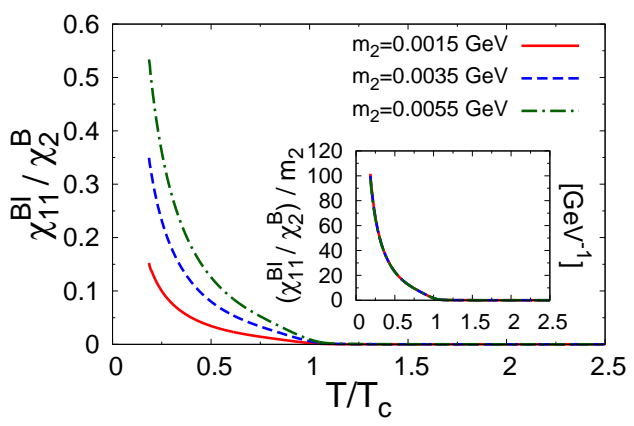

Figure 4: Ratio of $\mathrm{B}-\mathrm{I}$ correlation to baryon number fluctuation at $\mu_{B}=0$.

$$
m_{2}{ }^{\text {expt }}=\frac{\mathrm{R}_{2}^{\text {expt }}\left(T, \mu_{B}\right)}{\mathrm{R}_{2}^{\text {th }}\left(T, \mu_{B}\right)} \times m_{2}{ }^{\text {th }}
$$

where, 'expt' and 'th' denotes the experimentally measured and theoretically calculated values of the corresponding quantities respectively. To the best of our knowledge this is the first theoretical attempt which indicates that quark mass asymmetry in thermodynamic equilibrium can be directly measured from heavy-ion collision experiments.

\section{References}

[1] R. V. Gavai and S. Gupta, Phys. Rev. D66, 094510 (2002).

[2] G.M. de Divitiis et.al [RM123 collaboration], JHEP 1204, 124 (2012).

[3] R. Horsley, J. Najjar, Y. Nakamura, D. Pleiter, P.E.L. Rakow, G. Schierholz and J.M. Zanotti [QCDSF-UKQCD Collaboration], Phys. Rev. D86, 114511 (2012).

[4] A. G. Nicola, R. T. Andrés, Phys. Rev. D83, 076005 (2011).

[5] A. G. Nicola, R. T. Andrés, J. Phys. G:Nucl. Part. Phys.39 015004 (2012).

[6] T. Fujihara, T. Inagaki and D. Kimura, Prog. Theor. Phys. 117139 (2007).

[7] S. Mukherjee, M. G. Mustafa and R.Ray; Phys. Rev. D75, 094015, (2007).

[8] S.K. Ghosh, T.K. Mukherjee, M.G. Mustafa and R. Ray; Phys. Rev. D77, 094024, (2008).

[9] A. Bhattacharyya, S. K Ghosh, A. Lahiri, S. Majumder, S. Raha, and R. Ray; Phys. Rev. C89, 064905, (2014).

[10] S. Jeon and V. Koch, in Quark Gluon Plasma 3, Ed. R.C. Hwa and X. N. Wang, pg. 430 (World Scientific, Singapore, 2004), (arXiv:hep-ph/0304012).

[11] M. M. Aggarwal et. al., Phys. Rev. Lett. 105, 022302 (2010). 